\title{
Attention Deficits in Childhood-Onset Schizophrenia: Reaction Time Studies
}

\author{
Theodore P. Zahn, Leslie K. Jacobsen, Charles T. Gordon, Kathleen McKenna, \\ Jean A. Frazier, and Judith L. Rapoport \\ National Institute of Mental Health
}

\begin{abstract}
The hypothesis of continuity between childhood-onset and adult schizophrenia was tested by comparing the performance of 15 patients with childhood-onset schizophrenia and 52 age-matched controls on 2 reaction time paradigms that have been used to study adult schizophrenia. On simple reaction time to tones with regular and irregular preparatory intervals of 2,4 , and $8 \mathrm{~s}$, patients showed greater effects of the length of the preparatory interval in the regular condition and greater effects of the preparatory interval (girls only) and the preceding preparatory interval in the irregular series. On simple reaction time to random lights and tones, patients were faster on ipsimodal sequences than cross-modal sequences compared with controls. Overall, patients were much slower than controls in both paradigms. The results suggest similar attention dysfunction as is found in adult schizophrenia and thus are consistent with the continuity hypothesis.
\end{abstract}

Symptomatic schizophrenia typically develops in late adolescence to early adulthood, but some apparent cases have earlier onset, extending into the preadolescent period (Karno \& Norquist, 1989; Kramer, 1978). There has been some controversy about whether the childhood-onset disorder (defined here as having an onset at age 12 years or younger) is fundamentally the same disorder as the more common adult variety. Although child schizophrenia was once classified with infantile autism as a distinct class of disorders (American Psychiatric Association, 1968; Bender \& Faretra, 1972), careful studies of the phenomenology and clinical course of the disorder have indicated continuity between childhood- and adult-onset forms of the illness (J. R. Asarnow, 1994; Kolvin, Ounsted, Humphrey, \& McNey, 1971; Rutter, 1972; Werry, 1992). Although there is no definitive biological marker for schizophrenia, tests of the continuity hypothesis by studies of neuropsychological measures and biological markers that have statistically distinguished groups of adults with schizophrenia from normal controls and psychiatric contrast groups are still needed.

Several investigations using this general approach also supported continuity. Studies of brain morphology (Frazier et al.,

Theodore P. Zahn, Laboratory of Brain and Cognition, National Institute of Mental Health (NIMH); Leslie K. Jacobsen, Charles T. Gordon, Kathleen McKenna, Jean A. Frazier, and Judith L. Rapoport, Child Psychiatry Branch, NIMH. Charles T. Gordon is now at the University of Maryland School of Medicine. Kathleen McKenna is now at the Northwestern University School of Medicine. Jean A. Frazier is now at Harvard Medical School.

We thank Thalene T. Mallus for testing most of the participants and for data analysis.

Correspondence concerning this article should be addressed to Theodore P. Zahn, Laboratory of Brain and Cognition, NIMH, Building 10, Room 4C110, 10 Center Drive MSC 1366, Bethesda, Maryland 208921366. Electronic mail may be sent to ted_zahn@nih.gov.
1996), event-related potentials (Strandburg, Marsh, Brown, Asarnow, \& Guthrie, 1994), autonomic psychophysiology (Gordon et al., 1994; Zahn et al., 1997), and smooth-pursuit eye tracking (Jacobsen et al., 1996) have all found similar patterns for childhood- and adult-onset schizophrenia. Asarnow and colleagues reported similar deficits in childhood schizophrenia in attention and information processing as have been found in adult schizophrenia, revealed by the span of apprehension and other tasks (R. F. Asarnow et al., 1994; R. F. Asarnow \& Sherman, 1984). We report two further studies of attention in childhood-onset schizophrenia using reaction time (RT) paradigms, which have been used widely to study attention deficits in adult schizophrenia.

\section{Experiment 1}

Slow RT is one of the most, if not the most, consistent laboratory findings in schizophrenia research (Nuechterlein, 1977). However, because it is diagnostically nonspecific and possibly attributable to a generalized deficit in motivation secondary to the illness, its heuristic value is thought to be limited. For this reason, investigators have manipulated the parameters of the RT task to study more specific psychological processes. One such manipulation of a simple RT paradigm has been variations in the preparatory interval (PI), the interval between a warning signal and the RT stimulus. Trials with PIs of different lengths, ranging from $1 \mathrm{~s}$ to $25 \mathrm{~s}$ in some studies, are presented either in a regular series, in which the PI is constant for a series of trials, or in an irregular series, in which trials with the same PIs are presented in an unpredictable sequence. RT increases with increasing PI duration in the regular series and decreases with increases in PI duration in the irregular series. In schizophrenia, these relationships are typically exaggerated compared with controls ( see reviews by Nuechterlein, 1977, and by Rist \& Cohen, 1991).

The larger increase in RT with increases in the PI in the 
regular series may reflect a deficit in sustained attention, an inability to maintain a readiness to respond for more than a few seconds. Some, but not all, studies have observed a "crossover" pattern in schizophrenia patients in which, at the longer PIs ( $>4$ or $5 \mathrm{~s}$ ), RT is longer under regular conditions than under irregular conditions. Interpretations of crossover have included that the patients are unable to take advantage of the information provided by the regularity of the PI for more than a few seconds (Shakow, 1962), and that there is a "redundancy-associated deficit" in schizophrenia (Bellissimo \& Steffy, 1972).

The interpretation of larger decreases in RT with increasing PI length in the irregular series in schizophrenia has been influenced by the finding that RT is affected by the PI on the preceding trial (PPI) as well as by the current PI such that RT is retarded for PPI longer than PI relative to PPI shorter than or equal to PI, an effect that is also more pronounced in schizophrenics than in controls (Zahn, Rosenthal, \& Shakow, 1963). Because the shorter PIs are more likely to be preceded by longer PIs, their disproportionate effect on slowing RT in schizophrenia may be a direct result of the PPI effect. Thus, RT in schizophrenia seems more influenced by immediately preceding events than by the more general properties of the irregular sequence as a whole, in which each PI is equiprobable on each trial.

The only prior RT study on childhood-onset schizophrenia known us (Czudner \& Marshall, 1967) used six PIs ranging from 1 to $15 \mathrm{~s}$ and found greatly elevated RTs in their schizophrenia group but equal crossover in both patients and controls and also in a retarded comparison group, which showed only moderately elevated RTs. The schizophrenia patients actually showed a smaller difference between regular and irregular series than the controls. These results are quite different from those of the adult studies, but because the study was done in an era with different diagnostic customs and no diagnostic criteria are given, it is impossible to interpret the findings. Experiment 1 is an attempt to replicate the adult findings in a childhood-onset cohort.

\section{Method}

\section{Participants}

The total schizophrenia group consisted of 23 patients for whom the onset of schizophrenia was at age 12 or earlier. Only 9 patients ( 8 male and 1 female) were able to complete the task when medication free.' These patients had been off medication for a mean of 22 days (range =14-29 days). Most patients were given clinical trials of haloperidol or clozapine or both. To maximize the sample size, data collected during drug trials were used when drug-free data were not available. This added 6 patients ( 2 males and 4 females), all taking clozapine, for a total of 15 ( 10 males and 5 females), who are the participants in this report. ${ }^{2}$ Their mean age of onset of psychotic symptoms was $10.2 \pm 1.4$ years, and at the time of testing their mean age was $14.3 \pm 1.9$ years (range = 11-19 years). Consensus diagnoses using the Diagnostic and Statistical Manual of Mental Disorders (third edition, revised; DSM-III-R; American Psychiatric Association, 1987) criteria were made by two child psychiatrists on the basis of information from medical records and referring physicians, and from unstructured and structured interviews of each participant and parents. Portions of the Schedule for Affective Disorders and Schizophrenia for School-Age Children-Epidemiologic Version (Orvaschell, Tabrizi, \& Chambers, 1980) and the Diagnostic Interview for Children and Adolescents Revised (DICA-R; Reich \& Welner, 1988) were used. All patients had both positive and negative schizophrenic symptoms. Because they were recruited primarily for a clinical trial of clozapine, they were selected for a lack of clinical response to, or intolerance of, conventional neuroleptics. Other inclusion criteria included absence of active medical or neurological disease and a premorbid fullscale IQ greater than 70. Comorbid diagnoses included depressive disorders $(n=3)$, Asperger's syndrome $(n=2)$, obsessive-compulsive disorder $(n=2)$, and Tourette's syndrome ( $n=1)$. Further details about patient selection and characteristics are presented elsewhere (Frazier et al., 1994; Gordon et al., 1994; McKenna, Gordon, Lenane, Kaysen, \& Rapoport, 1994).

The 52 controls ( 30 male and 22 female; mean age $=14.5 \pm 1.9$ years, range $=11-18$ years) were recruited from the community and were paid for their participation. They were screened by the DICA (Herjanic \& Campbell, 1977) and the Diagnostic Interview for the Parents of Children and Adolescents (Herjanic \& Reich, 1982). Controls who met criteria for any disorder (current or past) on either of those scales were excluded. In addition, any history of an Axis I disorder, learning disability, or mental retardation in a first-degree relative, as determined by structured interviews, was exclusionary for this group.

Parents' informed consent and patients' informed assent were obtained for all participants. The study was approved by the National Institute of Mental Health Institutional Review Board.

\section{Apparatus and Procedure}

$R T$ task. In a simple warned RT task, participants were instructed that on each trial an amber warning light would come on and, when ready, they should depress a telegraph key (placed on the arm of the participant's chair) and keep it held down until a tone ("beep") sounded, at which time they should release the key as quickly as possible. They were told further that we were timing how fast they responded to the beep.

The PI was measured from the lime the key was depressed to the onset of the RT stimulus. When the key was depressed, the amber light went out. If the participant released the key prematurely, the amber light reappeared and the same PI was restarted with the next keypress. The RT stimulus was an $80-\mathrm{dB}\left(.0002\right.$ dynes $\left./ \mathrm{cm}^{2}\right), 1,000-\mathrm{Hz}$ tonc, which was terminated by the key release. The intertrial interval was $2 \mathrm{~s}$.

Just before this test, participants were given two nine-trial series with regular 4- and 8-s PIs while physiological variables were being recorded. That test was considered as constituting practice trials.

Participants were given 55 trials with PIs of 2, 4, and $8 \mathrm{~s}$. On the first 27 trials (regular series), the PI was constant for each 9-trial block. After each of the first two blocks, participants were told that the PI would be lengthened, and they were reminded to continue to respond to the beep as quickly as possible. ${ }^{3}$ On the last 28 trials (irregular series),

\footnotetext{
${ }^{1}$ The test was not attempted in 4 patients who, when off medication, were considered to be a danger to themselves or others. One of them was testable on the clozapine trial. Ten patients were unwilling or unable to do or complete the RT protocol while off medication because of uncooperativeness, disorganization, or inattention.

${ }^{2}$ Three patients who could not complete the task when unmedicated did complete it on the haloperidol trial, but their performance was very erratic and variable, suggesting poor cooperation for at least part of the time. They were all more cooperative on the clozapine trial, so that was the one used.

${ }^{3}$ Previous research has shown differential order effects of regular series PIs in schizophrenics and controls (Zahn, Rosenthal, \& Shakow, 1961). Because a large number of patients was not anticipated, it was believed that using different orders would unnecessarily complicate matters. The PIs were given in ascending order of length because it is easier
} 
the same three PIs were presented in a pseudorandom sequence such that each PI preceded each other PI, including itself, an equal number of times, with the further constraint that the same PI did not occur more than twice in succession. The first trial was not used because there was no PPI. Before this series, participants were told that before the beep there would sometimes be a short wait, sometimes a medium wait, and sometimes a long wait and that they would not know what to expect beforehand. They were again reminded to respond to the beep as quickly as possible.

Tests and rating scales. The Peabody Picture Vocabulary Test (PPVT; Dunn \& Dunn, 1981) was used as an estimate of current intellectual functioning. This gives a standard score with $M=100(S D=$ 15). Symptoms were rated by the extended (24-item) Brief Psychiatric Rating Scale (BPRS; Overall, Hollister, \& Pichot, 1967), the Scale for the Assessment of Negative Symptoms (SANS; Andreasen, 1983), and the Scale for the Assessment of Positive Symptoms (SAPS; Andreasen, 1984) on each treatment regimen usually during the same week as the RT test. Means and standard deviations for each scale are as follows: PPVT, $M=71.1(S D=20.9)$; BPRS, $M=68.3(S D=16.2)$; SANS, $M=61.1(S D=27.6)$; SAPS, $M=39.3(S D=17.1)$.

\section{Data Analyses}

The mean and median of the RTs for each PI in each series were the basic data. These were analyzed by Group (schizophrenia, control) $\times$ Sex $\times$ Sequence (regular PI, irregular PI) $\times$ PI $(2 \mathrm{~s}, 4 \mathrm{~s}, 8 \mathrm{~s})$ analyses of variance (ANOVAs); the last two factors were repeated measures. BMDP program 4V (Dixon, 1992) with equal weighting of the cell means was used. ${ }^{4}$ In the case of main effects and interactions involving the PI, the Huynh-Feldt epsilon correction to the degrees of freedom was used. Because the results for the mean and median were usually similar, those for just the median are presented, but mean data are presented if there is a substantive difference from the median. Planned tests of the simple effects of PI sequence and of sex were also done in the case of interactions involving those factors and group. For the irregular PI, RTs on trials when the PPI was less than, equal to, or greater than the PI were compared.

\section{Results}

\section{PI and Sequence Effects}

Figure 1 shows the overall results for girls and boys separately. The group difference in the mean of the median RT for each data point was larger for girls: Group $\times \operatorname{Sex}, F(1,63)=$ $12.89, p<.0007$, but both boys and girls were reliably slower than controls, $F(1,63)=20.32$ and 62.99 , respectively, $p$ s $<$ .0001 .

The increase in overall RT for the irregular series was greater in the patients: Group $\times$ Sequence, $F(1,63)=8.33, p<.006$, and there was a marginal interaction with sex, $F(1,63)=3.39$, $p<.08$. The critical interaction of Group $\times$ Sequence $\times$ PI depended on sex, as shown by the quadruple interaction, $F(2$, $126)=4.87, p<.02, \epsilon=.95$. Tests of simple effects for boys and girls showed that a significant Group $\times$ Sequence $\times$ PI interaction was obtained for just the girls, $F(2,126)=7.68$, $p<.0009$. Tests of simple effects for controls and patients

for participants to manage the task when the easiest condition comes first and because many adult studies used the ascending order. showed that the Sex $\times$ Sequence $\times$ PI interaction was significant only for the patients, $F(2,126)=8.13, p=.0006$. Thus, the schizophrenia girls differed from the other groups on the relationship of sequence and PI. An analysis of crossover, defined as the difference between median RT at the 8-s PI for regular minus irregular series, showed no diagnostic differences.

Both the linear and quadratic components of the overall interaction of sequence and PI showed interactions with sex, $F \mathrm{~s}$ ( 1 , $63)=4.44$ and $6.25, p s<.04$ and .02 , respectively. Simple effects tests for sex showed diagnostic group differences for only the girls: linear, $F(1,63)=10.23, p<.003$; quadratic, $F(1,63)=7.05, p=.01$. For the regular series, just the linear slope for the patients was greater than that of controls, $F(1$, $63)=6.22, p<.02$, and there were no interactions with sex for either trend. For the irregular series there were interactions with sex for both trends-linear, $F(1,63)=4.14, p<.05$; quadratic, $F(1,63)=8.01, p<.007-$ and group differences in both trends only for females, $F \mathrm{~s}(1,63)=6.84$ and 15.88 , $p \mathrm{~s}<.02$ and .0002 , respectively. In the schizophrenia group, females had a more negative linear slope than the males in the irregular series, $F(1,63)=4.13, p<.05$, and a larger quadratic trend as well, $F(1,63)=11.69, p<.002$.

Another question is whether the PI effects are predictable from RT level or whether they provide unique information about schizophrenia. This question has been raised with respect to PPI effects (i.e., the elevation in RT when PPI > PI is compared with PPI $\leq$ PI) by the Chapmans and their colleagues (Miller, Chapman, Chapman, \& Kwapil, 1993), but it is equally applicable to PI effects. These authors reanalyzed two studies of adult schizophrenia, and reported that significant group differences in the PPI effect were reduced to nonsignificance when the variance resulting from differences in RT level during the irregular series was adjusted for. However, it could be argued that using the total or mean RT for the same series for which slopes or difference scores are computed as an estimate of RT level in this case is questionable, because this sum will be influenced by whatever factor is responsible for the increment in RT produced by the PI or PPI effect in question over some hypothetical minimal level. Therefore, a correlation between this score and the difference score or slope would be expected even if the factor responsible for the increase was independent of the RT level. Miller et al. (1993) recognized this when they indicated it would "be better to design studies so that an independent measure of overall slowness is available" (p. 150). Because the present study used both regular and irregular series, such an independent (i.e., not sharing common variance) measure is available for each series. Thus, the mean RT during the irregular series was taken as an estimate of overall slowness in reanalyzing the RT slope for the regular PI, and RT during the regular series was used to adjust the irregular PI and PPI effects. These analyses were confined to linear slopes.

First, following Miller et al. (1993, p. 148), a "leverage

\footnotetext{
${ }^{4}$ The covariance of age with overall RT or for the slopes of the PI functions was not significant for either of the two diagnostic groups or for any of the Diagnosis $\times$ Sex subgroups. In addition, the groups did not differ in age. Therefore, covarying age in an analysis of covariance would be inappropriate.
} 

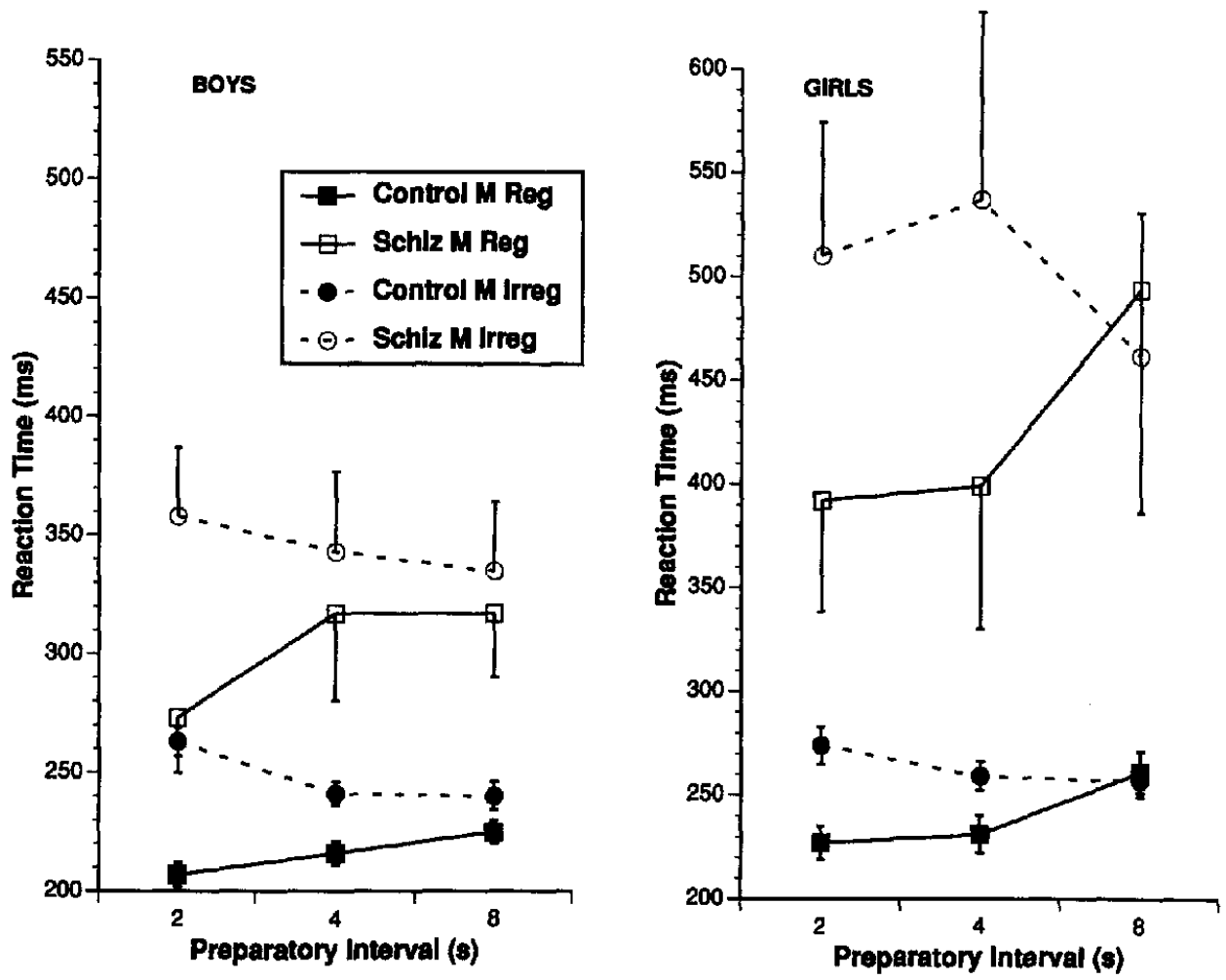

Figure l. Means of median reaction time as a function of regular (Reg) versus irregular (Irreg) sequence and preparatory interval for boys and girls in childhood-onset schizophrenia (Schiz) and control groups for Experiment 1.

analysis" was carried out to detect outliers in the control group that would seriously alter the correlations. The highest leverage score for either series was 0.26 , which is well below the 0.5 value cited by those authors as evidence of potentially spurious influence. The regression of mean RT on slope for the control group was used to compute residual scores for the slopes of all participants, as was done by Miller et al. (1993). An ANOVA on the residual scores for the slope of the regular series showed that the group effect was no longer significant $(F<1)$, showing that this effect is not independent of overall slowness. This reduction in significance is due to a correlation between the slope and RT mean of .29. For the irregular series, however, the correlation between the regular series mean RT and PI slope was only .01. The Group $\times$ Sex interaction remained significant, $F(1,63)=4.43, p<.04$, as did the effect for females only, $F(1,63)=7.70, p<.008$, showing that these effects are independent of overall RT.

\section{Effects of the Preceding PI}

Another set of analyses compared RTs during the irregular series when the PPI was less than, equal to, or greater than the current PI. As shown in Figure 2, RT increases across these conditions at a greater rate in the schizophrenia group. A Group $\times$ Sex ANOVA on the difference score of PPI longer than PI minus PPI shorter than PI showed a significant group effect,
$F(1,63)=13.15, p=.0006$, and no interaction with sex. An ANOVA of the residualized difference scores adjusted for the relationship with the mean regular series $\mathrm{RT}$ in the controls showed that diagnosis had an even more significant effect, $F=$ $25.70, p<.0001$, because of a correlation of -.14 between the difference score and mean RT. Thus, the PPI had a greater effect on RT in the schizophrenia group independent of their overall slowness.

\section{Discussion}

The results of this paradigm are similar to those reported in adult schizophrenia in several respects. First, RT was much slower in the patients. As mentioned earlier, this is an almost universal finding in adult schizophrenia research, but slow RT is considered a nonspecific marker of generalized dysfunction. Second, patients had a larger difference in overall RT between the regular and irregular series. Third, patients had a larger increase in regular series $\mathrm{RT}$ in relation to PI duration, although this was dependent on overall slowness. Fourth, the PPI versus PI effect was greater in the schizophrenia patients than in controls. This can be considered the most robust of the PI effects because it was independent of both sex and general slowness. In general, the patients were well within the range of values for RT level and PI effects reported in previous studies on adults (Rist \& Cohen, 1991). The groups were quantitatively different 


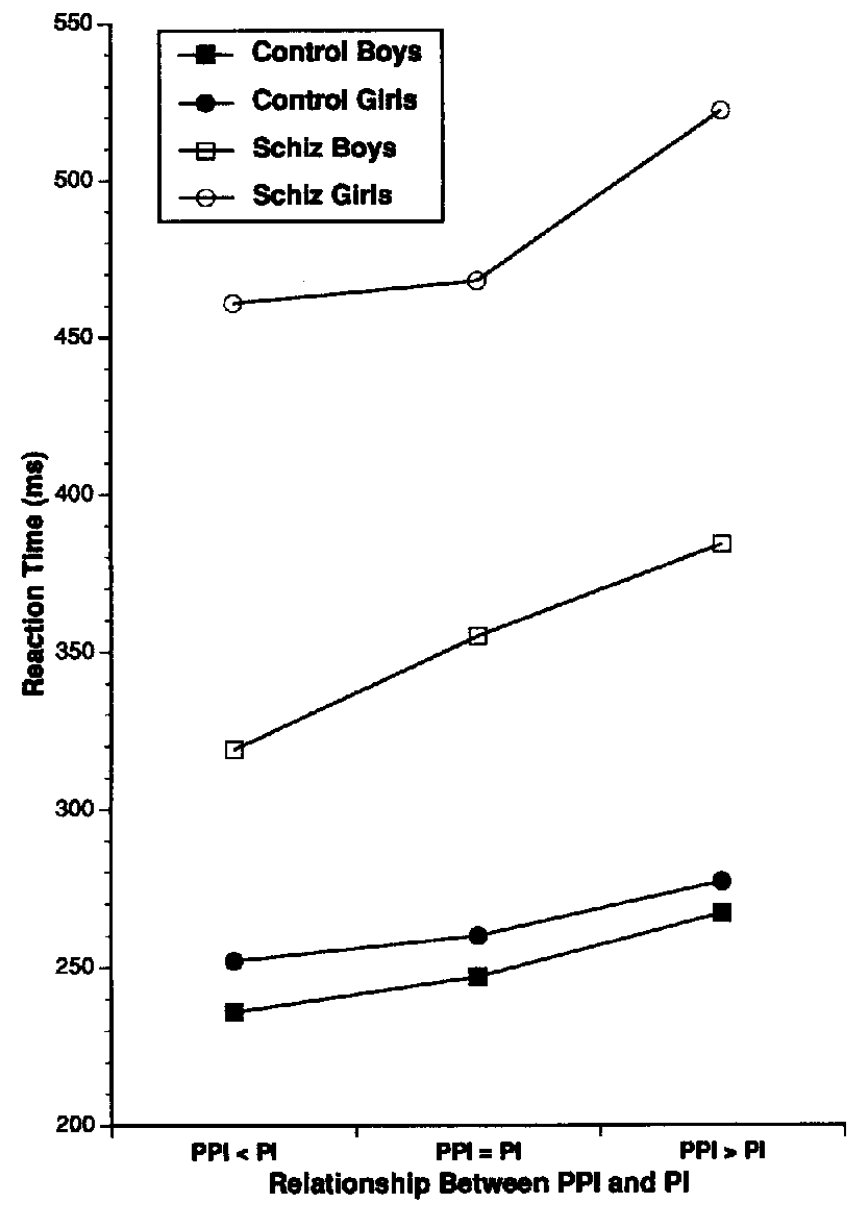

Figure 2. Means of median reaction time as a function of the relationship between the preceding preparatory interval (PPI) and the current preparatory interval (PI) for boys and girls in childhood-onset schizophrenia (Schiz) and control groups for Experiment 1.

in their PI and PPI effects, not qualitatively different, similar to results in adult samples, as pointed out by Rist and Cohen (1991).

However, there were also differences from adult studies. First, the greater negative slope in the irregular series in the schizophrenia group was due almost entirely to females. Studies of adult males with schizophrenia have clearly shown greater irregular PI effects compared with controls. Sex differences have been infrequently investigated..$^{5}$ In a study of acute patients, Zahn and Carpenter (1978) reported more females in a poor prognosis group who also had slower RT but not different PI or PPI slopes, nor were there sex differences in RT. In the present study, the female patients' scores on the PPVT were nonsignificantly lower, $t(13)=1.56$, and they were not rated as more symptomatic than the males on the BPRS, SANS, or SAPS, $t(13) \leq 0.50$, for the treatment on which the test was done. However, the girls had higher BPRS and SANS scores than the boys when the off-drug ratings were used for all participants, $t(13) \geq 2.17, p<.05 .^{6}$ It is not possible to determine whether this is typical of childhood-onset schizophrenia because the few previous studies have been heavily weighted with males and sex differences have not been reported (R. F. Asarnow et al., 1994; Czudner \& Marshall, 1967). However, because negative symptoms are indicative of poor prognosis, and poor prognosis in schizophrenia is associated with more impaired information processing (Nuechterlein, 1977; Zahn \& Carpenter, 1978), then the data are, to that extent, consistent with previous studies in adult schizophrenia. Low intellectual functioning, by itself, is unlikely to influence RT appreciably. Previous research has shown only minor elevations in RT level and not exaggerated PI effects in retarded persons (Czudner \& Marshall, 1967; Tizard \& Venables, 1956). However, the lower, albeit nonsignificant, PPVT scores in the girls, along with their increased offdrug higher negative symptom ratings, may indicate a more severe cognitive dysfunction, some of which at least may have persisted during drug treatment, as suggested in the next paragraph, and influenced RT performance.

Because 4 of the 5 girls were among the 6 patients tested while taking clozapine, this could have influenced the sex differences. However, comparison of unmedicated tests with clozapine tests on overall RT in the 9 patients with both tests showed no significant drug effect, paired $t(8)=0.42$, confirming a previous study on adults (Zahn, Pickar, \& Haier, 1994). In both studies, the major effect of clozapine was to increase the number of patients who were able to perform and complete the test. Prior studies of typical neuroleptics have also been unable to demonstrate effects on mean RT or its variation with the PI (Spohn \& Strauss, 1989). In addition, Goldberg et al. (1993) reported no effects of clozapine on a wide battery of neuropsychological tests. Thus, it is unlikely that the sex differences are due to a confound with medication status or that the data have been affected appreciably by the inclusion of data from some medicated patients.

A second difference from prior studies is that in irregular sequences schizophrenia groups frequently show a very sharp elevation in RT at the shortest PI length with a negatively accelerated decline as the PI increases (Nuechterlein, 1977; Rist \& Cohen, 1991). This was not observed here. Indeed, the schizophrenia girls showed just the opposite quadratic trend. Although in most previous studies the shortest PI was $1 \mathrm{~s}$ rather than the $2 \mathrm{~s}$ used here, this cannot explain the present data. The female patients showed small differences between the 2-s and 4-s PIs for both regular and irregular series, so perhaps their appreciation of elapsed time was defective at short intervals.

\footnotetext{
${ }^{5}$ An informal review of many of the major articles on RT in schizophrenia showed that, even when both sexes have been included, it is rarely mentioned that sex differences have been analyzed. Although slower RT in females with schizophrenia than in males has been reported, at least under some conditions (Karras, 1968; Rosenbaum, 1967), the typicality of this finding cannot be determined. Gruzelier (1991) reported inferior performance by female schizophrenia patients compared with males on a number of neuropsychological tests, which he felt tapped mainly right parietal lobe functioning, but not on tasks that were mediated presumably by other brain areas.

${ }^{6}$ For the entire sample, including those who could not do the task under any condition, the girls scored higher on the SANS, $t(20)=2.62$, $p<.02$, and lower on the PPVT, $t(21)=2.17, p<.05$, but they were not different on the BPRS or SAPS
} 
A third difference is the absence of significant crossover in the schizophrenia group. This could be due to the generally elevated irregular RT in comparison to regular RT in the patients. It seemed possible that, because the irregular series was given after the regular series, these young patients showed restlessness or fatigue, which manifested itself primarily on long PI trials. However, the means of median RT for the first half and the last half of the irregular series - which had very similar PI distributions-were almost identical: mean difference $=0.3$ $\mathrm{ms}, t(14)=0.03$, suggesting that fatigue did not play a major role. Many adult studies using this paradigm, particularly those for which the longest PI is less than or equal to $10 \mathrm{~s}$, have not reported much ( $\geq 20 \mathrm{~ms}$ ) crossover in schizophrenia samples (Rist \& Cohen, 1991). Thus, perhaps the present result is not too atypical.

\section{Experiment 2}

In contrast to the previous simple RT paradigm, which can be thought to measure sustained attention, a second paradigm measured RTs to lights and tones under three conditions: regular, in which blocks of all lights and all tones were given; choice, in which the two stimuli were presented randomly, lights being responded to with one hand and tones with the other; and "cross-modal" (CM), in which the two stimuli were presented randomly but each was responded to with the same (preferred) hand. The CM condition differs from the regular condition in that there is stimulus uncertainty, whereas the choice condition additionally requires response selection. Thus, one aim of this experiment was to study diagnostic differences in these two aspects of selective attention. In addition, paradigms in which there is stimulus uncertainty have been used to study modality shift effects in schizophrenia by comparing RT on trials in which the preceding stimulus is in the same modality (ipsimodal) with RT on trials in which the preceding stimulus is in the other modality (cross-modal). Although the literature is conflicting on whether there is a greater deficit in choice than simple RT in schizophrenia, the original finding of greater retardation in RT on cross-modal compared with ipsimodal trials (Sutton, Hakerem, Zubin, \& Portnoy, 1961) has usually been replicated (Nuechterlein, 1977; Rist \& Cohen, 1991).

\section{Method}

\section{Participants}

As in Experiment 1, some schizophrenia patients did not complete the test or gave erratic and variable performance because of a failure to cooperate under some conditions. The data for 15 patients $(9$ males and 6 females) are included: 10 were off drugs, 3 were taking haloperidol, and 2 were taking clozapine. The patients were the same as for Experiment 1 with two exceptions: 1 boy was excluded because of lack of cooperation on this paradigm, and 1 girl who could not manage the other paradigm was able to do this one. In addition, 5 patients were tested on a different medication. The controls were the same as in Experiment 1.

\section{Apparatus and Procedure}

Keypress RTs to lights and tones were recorded. The response apparatus consisted of two lever switches mounted on a board placed across the arms of the participant's chair, one switch conveniently placed for operation with each hand. Tones $(1000 \mathrm{~Hz})$ were presented from a speaker on the floor in front of the participant. The light source was a green electroluminescent lamp (Grimes Mfg. Co. Urbana, Ohio) with an advertised rise time of $90 \mu$. This lamp is a square panel- $165 \mathrm{~mm}$ on all sides-but only the central $6.25 \mathrm{~cm}^{2}$ was exposed. It was put inside a hood to attenuate ambient light and placed at eye level $125 \mathrm{~cm}$ in front of each participant. Tones and lights were of 50-ms duration and had been equated for subjective intensity in a separate group of adults. Intertrial intervals were random between 2 and $3 \mathrm{~s}$.

Participants were first given regular series of 10 tones and 10 lights (Reg1), each preceded by at least four practice trials. Then 49 choice RT trials were given in which the tones and lights were presented in a pseudorandom sequence, the same for all participants. Alternate participants responded with the right hand to tones, and with the left hand to lights; for the remainder, the converse stimulus-response (S-R) mapping was used. This was followed by the CM paradigm, which also consisted of a pseudorandom series of 49 lights and tones, but the participant responded to both stimuli with the preferred hand. Finally, regular series of 10 lights and 10 tones (Reg2) were given. Participants responded to these with the preferred hand.

Because the speaker for the tone presentation was inadvertently placed about $10^{\circ}$ off center to the left, the $S-R$ mapping variable provides a test of S-R compatibility on RTs; the tone-left hand mapping should produce faster RTs than the opposite mapping.

\section{Results}

\section{Effects of Conditions, Stimuli, and Mapping}

For analyses that include choice RT, participants making more than 11 errors were excluded, ${ }^{7}$ and RTs for only correct-response trials were included. One control and 2 schizophrenia patients were excluded for this reason. Figure 3 shows the basic results across the four conditions for the two stimuli. A Group $\times \operatorname{Sex}$ $\times$ Condition $\times$ Stimulus ANOVA ${ }^{8}$ on median RT showed a group effect, $F(1,59)=36.57, p<.0001$, and a Group $\times$ Condition $\times$ Stimulus interaction, $F(3,177)=7.02, p=.001$, $\epsilon=.71$. Tests of simple effects for each stimulus, however, showed significant Group $\times$ Conditions terms for both tones, $F(3,177)=4.07, p<.02, \epsilon=.78$, and lights, $F(3,177)=$ $4.54, p=.01$. Although girls had slower RTs than boys, $F(1,59)$ $=7.07, p=.01$, sex did not interact with any other dimension, including group. To probe the interactions involving Group $\times$ Condition, ANOVAs contrasting choice and CM conditions with the combined regular series and with each other were carried out.

For choice versus regular series RT, a Group $\times$ Condition interaction reflected a greater increment in RT for the choice condition in the patients, $F(1,60)=10.82, p<.002$. This was marginally affected by S-R mapping: Group $\times$ Condition $\times$ Map, $F(1,60)=3.76, p<.06$. The patients showed a light

\footnotetext{
${ }^{7}$ This number was chosen partly on the basis of the distribution of error scores and partly because $25 \%$ or more errors seemed too high to accept.

${ }^{8}$ Preliminary analyses of the regression of age on median RT for the four conditions and for the grand mean RT for males and females for each diagnosis showed that age was not significantly correlated with RT for any group under any condition, so ANOVAs were used in the main analyses.
} 


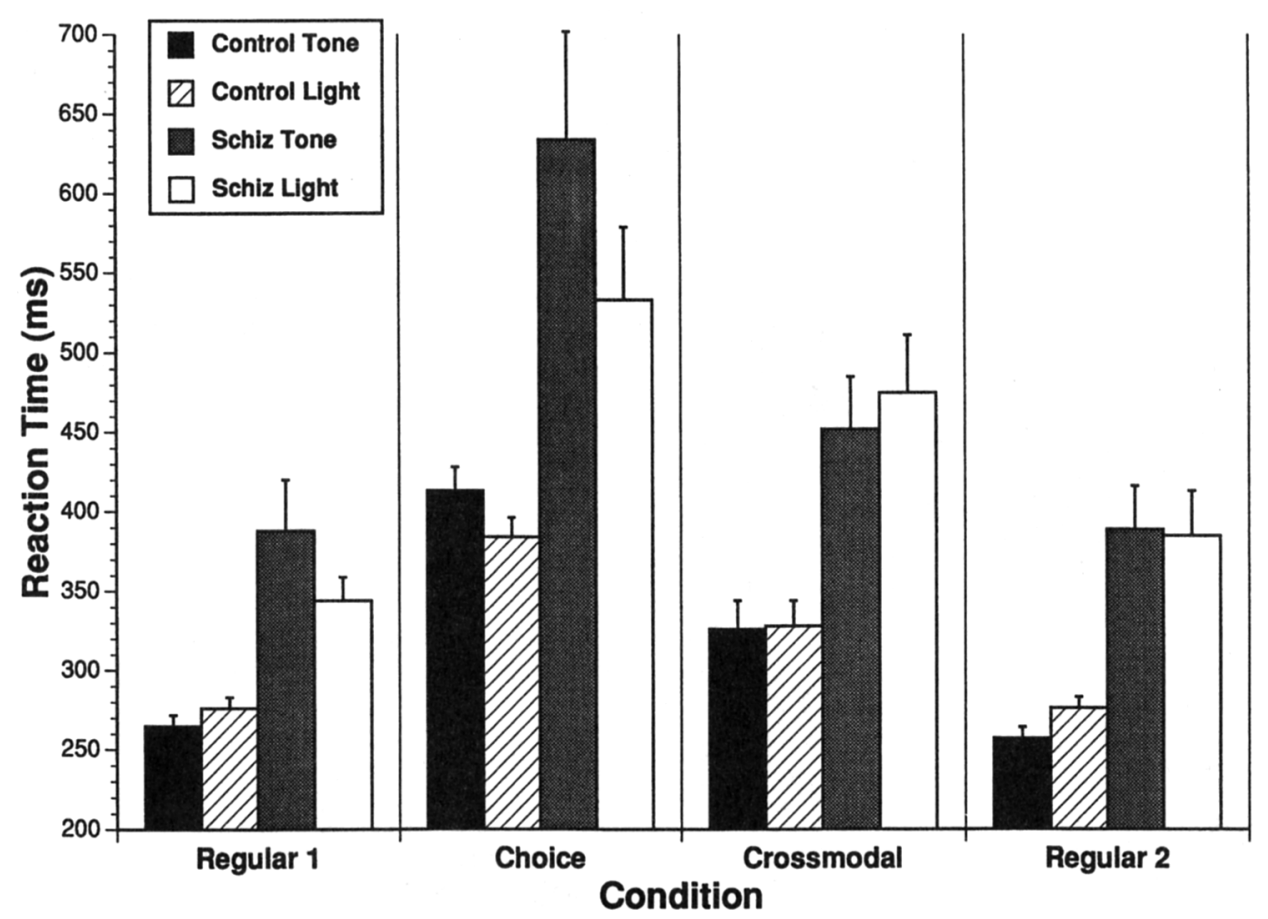

Figure 3. Means and standard errors of median reaction time for childhood-onset schizophrenia (Schiz) and control groups to tone and light stimuli for regular, choice, and cross-modal conditions in Experiment 2.

advantage ( see Figure 3), Group $\times$ Stimulus, $F(1,60)=10.77$, $p<.002$. For choice RT alone, RT was faster for the tone-left hand mapping (see Figure 5 ), $F(1,60)=24.63, p<.0001$. This was greater in the schizophrenia group: Group $\times$ Map, $F(1,60)=4.87, p<.04$, and marginally greater for the tone: Group $\times$ Map $\times$ Stimulus, $F(1,60)=3.79, p<.06$. The mapping effect was greater in the patients for both stimuli but significantly only for the tone, $F(1,60)=5.95, p<.02$. Because the speaker for the tone was slightly left of center, these analyses indicate an S-R compatibility effect that was larger in the patients.

The overall increment in RT in the $\mathrm{CM}$ condition versus regular series was not differentially affected by group. However, there was a marked interaction with stimulus, $F(1,64)=17.17$, $p=.0001$. Simple effects tests for tones and lights showed that the increment in CM RT for the patients was greater than that in controls for lights, $F(1,64)=5.15, p<.03$, but not for tones.

Contrasting the choice and CM conditions also failed to show an overall group difference, but there was an interaction with stimulus, $F(1,59)=16.75, p=.0001$. The patients showed a greater increment than controls in choice RT for tones, $F(1$, $59)=6.72, p<.02$, but not for lights ( see Figure 3 ).

\section{Crossmodal Versus Ipsimodal Sequence Effects}

The effects of the sequence of tone-light stimuli in both the choice RT and cross-modal RT paradigms were examined by comparing the difference in RTs for cross-modal minus ipsimodal sequences. In contrast to the controls, the schizophrenia group showed cross-modal retardation for both stimuli in the CM condition and for tones but not lights for choice RT (Figure 4). ANOVAs for each condition showed significant differences for means but not for medians, although the differences were in the same direction. For means of choice RT, a Group $\times$ Stimulus effect, $F(1,62)=8.60, p<.005$, was due mainly to the group difference for the tone, $F(1,62)=4.09, p<.05$. For the CM condition, the main effect for group, $F(1,64)=$ $7.82, p<.007$, reflected the relative cross-modal retardation for both stimuli by the patient group.

\section{Error RTS Versus Correct RTs}

The controls averaged $1.9 \pm 2.3$ errors in the choice condition and the patients averaged $2.72 \pm 2.6$. This difference does not approach significance, nor were there any interactions with stimulus or mapping. The RTs of correct responses and of error responses were compared for the 50 controls and 12 patients who had at least one error. As seen in Figure 5, error RTs were faster than RTs of correct responses for both groups, especially in the schizophrenics. However, the group difference depended on mapping as shown by a Group $\times$ Condition $\times$ Map interaction, $F(1,58)=6.77, p<.02$, and a significant Group $\times$ Condition effect for just the less compatible light-left hand condition, $F(1,58)=22.99, p<.0001$. There were no group differences on just the error RTs (although for the RT means, $p$ 


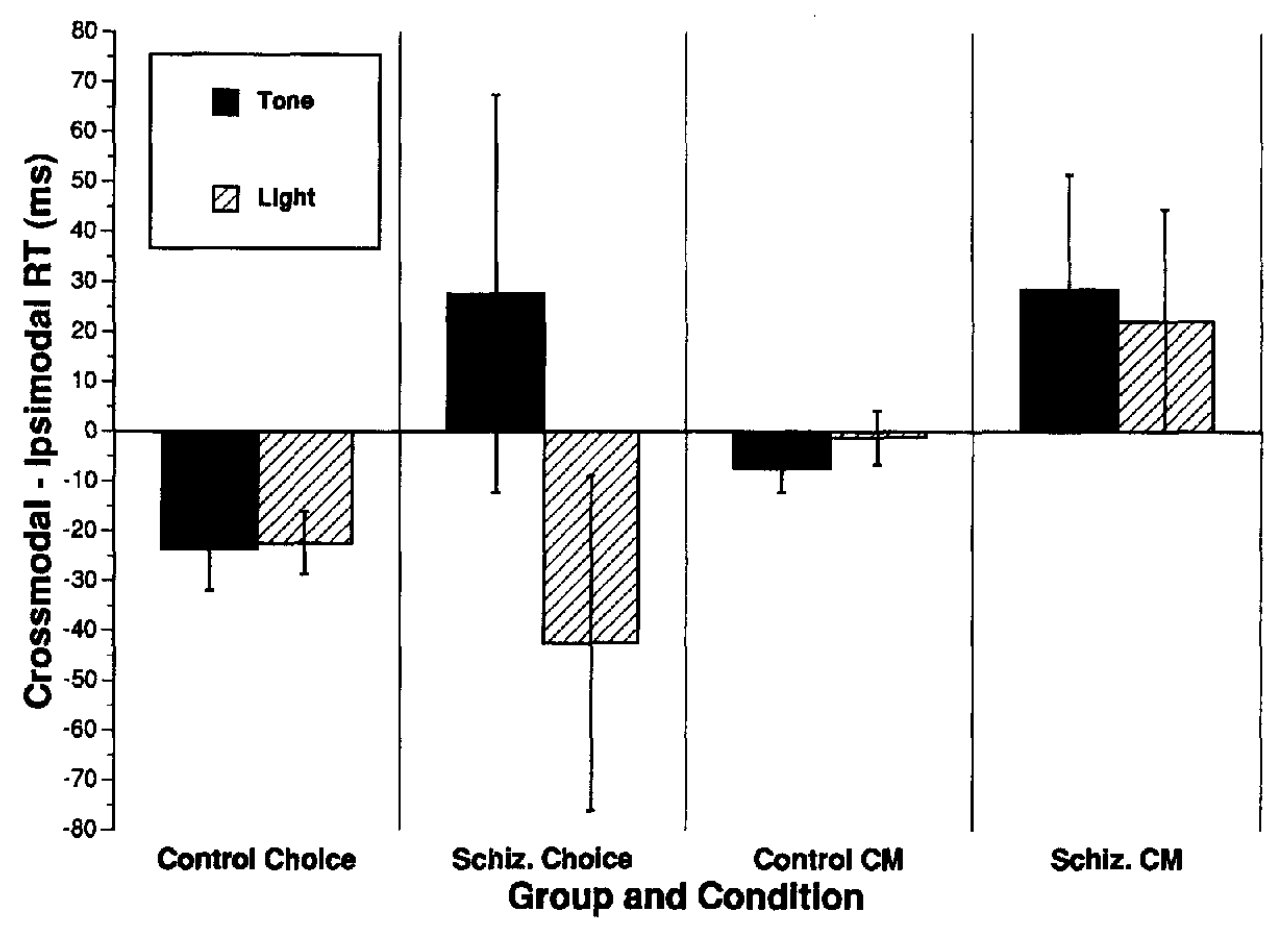

Figure 4. Means and standard errors of differences in reaction time (RT) means of cross-modal and ipsimodal sequences for childhood-onset schizophrenia (Schiz.) and control groups to tone and light stimuli in the choice and cross-modal (CM) conditions in Experiment 2.

$<.06)$ and no interactions with mapping, suggesting that the Group $\times$ Condition interaction was due to differences in the RTs on correct trials. However, compared with the grand mean of the two regular series procedures, error RTs were much slower in controls $(351 \pm 87$ ys. $267 \pm 47), F(1,60)=113.3, p<$ .0001 , but only slightly slower in the patients $(397 \pm 49$ vs. $371 \pm 78, p=n s)$. The Group $\times$ Condition interaction here was significant, $F(1,60)=10.36, p<.003$.

\section{Discussion}

The schizophrenia group showed a greater increment than controls in choice RT compared with constant-stimulus (regular) RT. They did not show a greater deficit in the CM condition overall, but did so when the stimulus was the light. Both choice and $\mathrm{CM}$ paradigms involve random stimulus presentation-or stimulus uncertainty - but choice RT, in addition, requires response selection. The data are thus consistent with the hypothesis that impairment in schizophrenia is greater as a function of the number of operations required for a response. Moreover, greater slowing was produced by incompatible $S-R$ mapping in the patients, and this was more pronounced to tone stimuli. This probably contributed to the greater deficit in choice compared with CM RT for just the tone in the patients. The lack of a group difference in errors suggests that participants in the two groups were cqually likely to adopt an "accuracy set" as opposed to a "speed set." This came at a greater cost in time for the schizophrenia participants.
Although there are conflicting findings in the adult schizophrenia literature on choice versus simple RT (Nuechterlein, 1977), it has been pointed out that most of the studies have used spatial choice RT in which there is high S-R compatibility (Zahn, 1977). The present finding that the patients were more affected by that variable suggests that comparisons with the spatial RT studies may not be valid. Our S-R compatibility finding, however, is consistent with a finding in adult schizophrenia (Karras, 1973).

The reasons for the modality differences in these effects are not clear. One possibly relevant factor is visual dominance. Studies of choice RT using probes of simultanenus presentation of visual and auditory stimuli have established that there is a preferential bias for attention to be directed to the visual channel when stimulus modality is uncertain (Colavita, 1974; Egeth \& Sager, 1977; Posner, Nissen, \& Klein, 1976). Visual dominance also occurs in schizophrenia (Zahn et al., 1994). The longer RT and cross-modal retardation to tones shown by the patients in the choice paradigm compared with those of controls thus suggest greater slowness in switching attention to the nonpreferred modality.

A study on adult schizophrenia comparing simple RT to constant and variable tone-light sequences, similar to the present CM condition, reported a greater increment in RT in the variable-stimulus condition in schizophrenia for both stimuli, and this was larger for the tone (Kristofferson, 1967). Despite some differences in method, the present study partially replicates that 


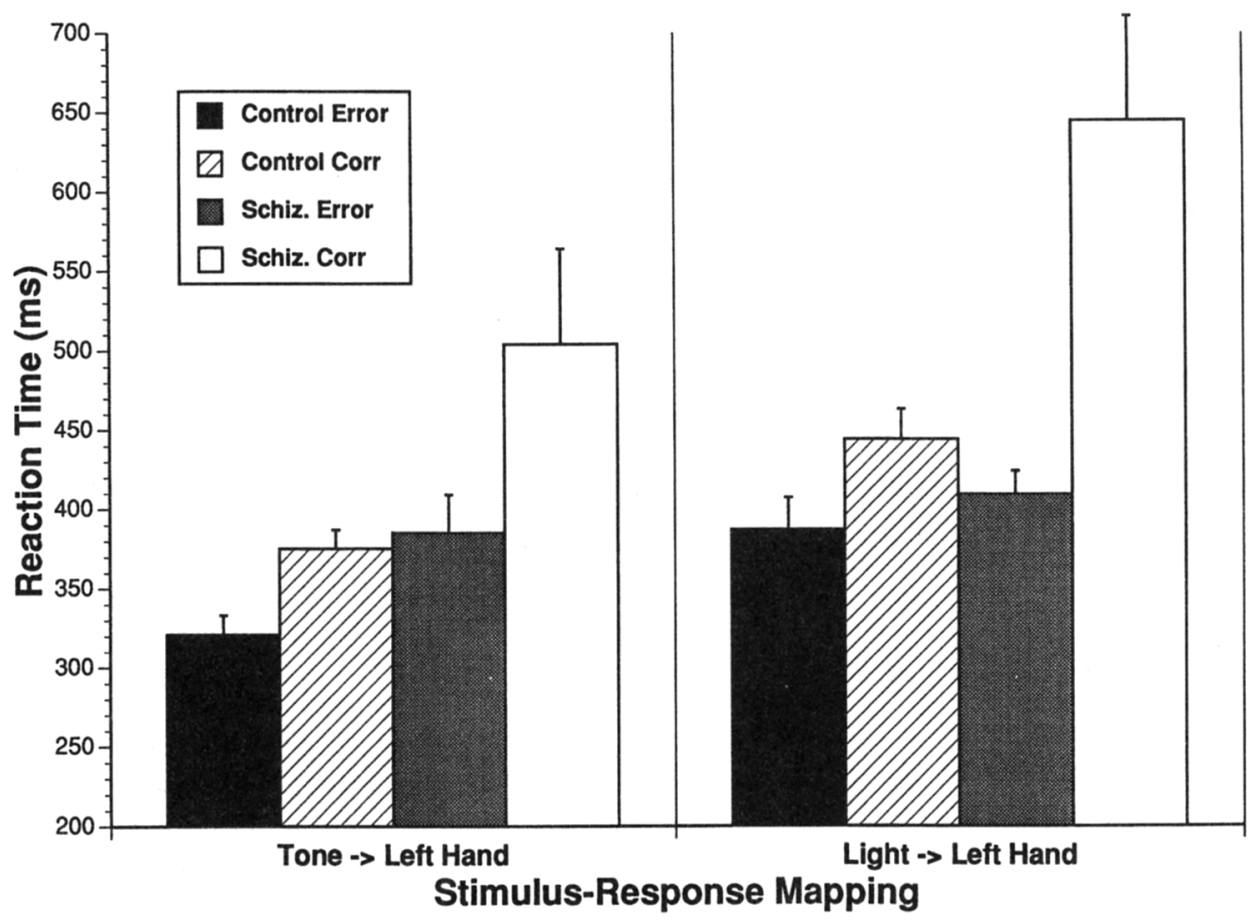

Figure 5. Means and standard errors of median reaction time on error trials and correct response (Corr) trials for childhood-onset schizophrenia (Schiz.) and control groups in the choice procedure for participants who responded to the tone with the left hand or to the light with the left hand for Experiment 2 .

one, but the group difference was significant only for lights. The present result may be partly due to the atypical relative slowness to tones for the first regular condition in the patients, because it is well established that under constant-stimulus conditions RT to tones is faster than to lights at moderate intensities (Woodworth \& Schlosberg, 1954). However, Kristofferson (1967) also reported faster constant-stimulus RTs for lights than for tones in patients and the reverse in controls, but this interaction was not tested for significance.

Most of the previous schizophrenia research using a CM paradigm has focused on comparing cross-modal and ipsimodal sequences. The present finding of a greater ipsimodal advantage for the schizophrenia group in the CM condition is similar to previous findings in adult schizophrenia (Nuechterlein, 1977; Rist \& Cohen, 1991). It may be noted that our finding is partly due to a small cross-modal advantage in controls. This is not too different from previous studies, however. Of eight studies summarized by Rist and Cohen (1991, p. 258 ) involving nine control groups for tone stimuli, only three reported cross-modal slowing greater than $10 \mathrm{~ms}$. For lightimperative stimuli, the comparable figure was two of seven. However, most studies reported cross-modal slowing in schizophrenia for tones of longer than $30 \mathrm{~ms}$, which is greater than the present study. The cross-modal slowing for lights is less; only two of nine groups cited exceeded the 22-ms value of the present study. This was almost always less than the effect in the tone data but not always significantly so. The present results are slightly in the same direction. The overall difference between patients and controls is well within the range of adult studies (Rist \& Cohen, 1991). There were no significant modality shift effects in RT medians. Although this weakens the generality of the results, previous studies have reported only means.

Sequence effects for choice RT in schizophrenia have not been studied as extensively. The greater cross-modal retardation in childhood-onset schizophrenia than in controls only for tones in the choice condition partially replicates a study on adults by Sutton and Zubin (1965, Experiment B), but those investigators found the effect only for males and not for females. The modality difference is also consistent with that found in adult data from CM paradigms. Whether the enhanced visual dominance shown by the schizophrenia group in choice RT had a role in this effect is problematic. One can see how an ipsimodal tone sequence might reduce RT by overcoming the "natural" bias to attend to the visual channel, but this line of reasoning does not explain the cross-modal advantage shown by the patients to the light or by the controls to both stimuli.

The marked reduction in RT on error trials compared with that on correct trials for both groups suggests that the errors are impulsive in nature rather than random lapses in attention. However, error RT in the schizophrenia participants was almost at their regular series level, whereas for the controls it was significantly higher (and had a higher variance). This suggests that in the controls on some trials there may have been either lapses of attention or some attempt at error correction, leading to relatively longer RTs. In contrast, in the patients, virtually all errors were impulsive and possibly unrecognized. 


\section{General Discussion}

Because the present participants were chosen for a lack of response to or inability to tolerate conventional neuroleptic drugs and were diagnosed using a rather narrow definition of schizophrenia (Gordon et al., 1994), they might be thought to be more cognitively impaired than an unselected pediatric group with schizophrenia. However, the most disorganized patients were unable to do these procedures, so the patients who were able to complete the tests may be closer to average. Attention deficits in unselected samples of childhood-onset schizophrenia using different techniques have been reported (R. F. Asarnow et al., 1994; R. F. Asarnow \& Sherman, 1984). In view of the high significance levels of most of the differences reported here, it is difficult to imagine that they would disappear completely in tests of less selective samples of this population, although they might be reduced in magnitude.

Although there were some exceptions, the findings in general were similar to those reported in previous studies on adult schizophrenia. The present study thus provides further confirmation of the hypothesis of continuity of childhood-onset and adolescent/adult-onset schizophrenia.

What do these similarities tell us about the abnormalities common to childhood- and adult-onset schizophrenia? The most striking abnormality is the considerably longer RT under all conditions in the schizophrenia group. During a prior session in which physiological recording was done, the patients showed marked impairment in electrodermal orienting responses to nonsignal tones and RT stimuli and impaired heart rate decelerations during an 8-s PI (Zahn et al., 1997). As with impairments in event-related potentials (Strandburg et al., 1994), these findings suggest an impairment in mobilizing processing resources (Dawson, 1990; Öhman, 1979). The improvement in warned RT in relation to unwarned RT has been attributed to the fixed warning interval enabling a state of maximal preparation to respond (Niemi \& Näätänen, 1981). Therefore, the deficit in effortful processing suggested by the physiological data may manifest itself in a simple RT task as deficient peak preparation (Shakow, 1962). Increases in RT in relation to PI duration have been attributed to greater difficulties in timing peak preparation at longer PIs (Niemi \& Näätänen, 1981). The disappearance of the group difference in the slope of the regular RT curve when the slopes were adjusted for differences in slowness suggests that both the overall RT deficit and the greater slope are determined, at least in part, by similar deficiencies in preparation. In Shakow's (1962) terms, adopting a major "set-to-respond" and maintaining it seem to be related processes.

Two contemporary theories seem to account for differences in preparation. One is Cohen and Servan-Schreiber's (1992) hypothesis that a fundamental problem in schizophrenia is the inadequate representation of context for the control of action, which they attribute in part to disturbances of prefrontal cortex and mesocortical dopamine functioning. The role of dopamine activity may be questioned on the basis of the lack of effects of neuroleptic drugs, which alter it, on RT and cognitive tests. In other respects, this theory is similar to a second theory, that of Goldman-Rakic (1994), who hypothesized that many of the cognitive problems in schizophrenia are due to a deficiency of working memory, which is related to impaired frontal lobe function. Working memory and the representation of context seem closely allied to the concept of preparation in that the internalized instruction or set to move one's finger as quickly as possible at the onset of a stimulus is kept "close to the surface" by this function. Goldman-Rakic hypothesized that "prefrontal cortex 'accesses' and holds 'on line' representational knowledge of the outside world" $(1994$, p. 355). Rist and Cohen pointed out that preparation involves "motor programming and initiation closely linked to frontal lobe functioning" (1991, p. 254). When this is inadequate, the appropriate behavior does not fail to occur, it just occurs after a longer delay because some of the "preparation" now must be done after the onset of the stimulus. Such a mechanism thus may account in part for slow RT in general in schizophrenia. Because working memory fades over the passage of time on a scale of seconds (Goldman-Rakic, 1994), the greater PI effect in the regular series in the schizophrenia group might indicate a less firmly established or more quickly fading memory trace.

To account for irregular PI and PPI effects, Niemi and Näätänen (1981) suggested that expectancy of PI length may be modified by the preceding PI. That differences in expectancy per se are responsible for the differences in PI and PPI effects is unlikely because, even when participants are informed of the PI length beforehand, the greater PI and PPI effects in schizophrenia may remain (Zahn, 1970). Another process suggested by Niemi and Näätänen ( 1981) is "repreparation," in which, when there are a few discrete PIs, participants attempt to reach peak preparation at appropriate times during the PI. This involves memory of the time intervals and time estimation, both of which may be influenced by PPI duration, particularly at short PIs. Using this model, the deficit in schizophrenia could come from a greater effect of PPI duration on time estimation or a deficit in switching attention to the RT stimulus when preparation is minimal.

The detrimental influence of long PPIs in schizophrenia has also been attributed to their inordinate effect on preparation at the expense of a more adaptive strategy of basing preparation on the properties of the sequence as a whole (Zahn et al., 1963) or to minor sets interfering with a major set (Shakow, 1962). A similar argument could pertain to the detrimental influence that a light stimulus on one trial has on RT to a tone on the next trial, as shown in the greater cross-modal retardation in schizophrenia. In terms of the hypotheses of deficiencies in the internal representation of context or in working memory in schizophrenia, the more robust internal representations of normal persons should be less subject to distortion or weakening by transient context effects. Choice RT, which requires response selection, and S-R incompatibility both produced additional RT deficits in schizophrenia. They seem to put more demands on working memory or require more complex internal representations.

Thus, deficiencies in the internal representation of context and in working memory seem capable of explaining most of the major findings of this study. Conceptually, these ideas are not dissimilar to Shakow's (1962) segmental set hypothesis, but they have the great advantage of being based on neurophysiological evidence or network models that are independent of the 
data on schizophrenia. This study was not designed to provide a critical test of those models, however, and until such studies are devised, the fit of the models to schizophrenia RT data should be considered tentative.

\section{References}

American Psychiatric Association. (1968). Diagnostic and statistical manual of mental disorders (2nd ed.). Washington, DC: Author.

American Psychiatric Association. (1987). Diagnostic and statistical manual of mental disorders (3rd ed., rev.). Washington, DC: Author.

Andreasen, N. C. (1983). The Scale for the Assessment of Negative Symptoms ( SANS). Lowa City, IA: University of lowa.

Andreasen, N.C. (1984). The Scale for the Assessment of Positive Symptoms (SAPS). Iowa City, IA: University of Iowa.

Asarnow, J. R. (1994). Annotation: Childhood-onset schizophrenia. Journal of Child Psychology and Psychiatry, 35, 1345-1371.

Asarnow, R. F., Asamen, J., Granholm, E., Sherman, T., Watkins, J. M., \& Williams, M. E. ( 1994) . Cognitive/neuropsychological studies of children with a schizophrenic disorder. Schizophrenia Bulletin, 20, 647-669.

Asarnow, R. F., \& Sherman, T. (1984). Studies of visual information processing in schizophrenic children. Child Development, 55, 249 261.

Bellissimo, A., \& Steffy, R. A. (1972). Redundancy-associated deficit in schizophrenic reaction time performance. Journal of Abnormal Psychology, 80, 299-307.

Bender, L., \& Faretra, G. (1972). The relationship between childhood schizophrenia and adult schizophrenia. In A. R. Kaplan (Ed.), Genetic factors in schizophrenia (pp. 28-64). Springfield, IL: Charles C Thomas.

Cohen, J. D., \& Servan-Schreiber, D. (1992). Context, cortex, and dopamine: A connectionist approach to behavior and biology in schizophrenia. Psychological Review, 99, 45-77.

Colavita, F. B. ( 1974). Human sensory dominance. Perception and Psychophysics, 16, 409 412.

Czudner, G., \& Marshall, M. (1967). Simple reaction time in schizophrenic, retarded, and normal children under regular and irregular preparatory interval conditions. Canadian Journal of Psychology, 21, $369-380$.

Dawson, M. E. (1990). Psychophysiology at the interface of clinical science, cognitive science, and neuroscience. Psychophysiology, 27, $243-255$

Dixon, W. J. ( 1992). BMDP statistical software manual. Berkeley, CA: University of Califomia Press.

Dunn, L. M., \& Dunn, L. M. (1981). The Manual of Peabody Picture Vocabulary Test-Revised. Circle Pines, MN: American Guidance Service.

Egeth, H. E., \& Sager, L. C. (1977). On the locus of visual dominance. Perception and Psychophysics, 22, 77-86.

Frazier, J. A., Giedd, J. N., Hamburger, S. D., Albus, K. E., Kaysen, D., Vaituzis, A. C., Rajapakse, J. C., Lenane, M. C., McKenna, K., Jacobsen, L. K., Gordon, C. T., Breicr, A., \& Rapoport, J. L. (1996). Brain anatomic magnetic resonance imaging in childhood onset schizophrenia. Archives of General Psychiatry, 53, 617-624.

Frazier, J. A., Gordon, C. T., McKenna, K., Lenane, M. C., Jih, D., \& Rapoport, J. L. (1994). An open trial of clozapine in 11 adolescents with childhood-onset schizophrenia. Journal of the Academy of Child and Adolescent Psychiatry, 33, 658-663.

Goldberg, T. E., Greenberg, R. D., Griffin, S. J., Gold, J. M., Kleinman, J. E., Pickar, D., Schulz, S. C., \& Weinberger, D. R. (1993). The impact of clozapine on cognition and psychiatric symptoms in patients with schizophrenia. British Journal of Psychiatry, 162, 43-48.
Goldman-Rakic, P. S. (1994). Working memory dysfunction in schizophrenia. Journal of Neuropsychiatry, 6, 348-357.

Gordon, C. T., Frazier, J. A., McKenna, K., Giedd, J., Zametkin, A., Zahn, T., Hommer, D., Hong, W., Kaysen, D., Albus, K. E., \& Rapoport, J. L. (1994). Childhood-onset schizophrenia: An NIMH study in progress. Schizophrenia Bulletin, 20,697-712.

Gruzelier, J. (1991). Hemispheric imbalance: Syndromes of schizophrenia, premorbid personality, and neurodevelopmental influences. In S. R. Steinhauer, J. H. Gruzelier, \& J. Zubin (Eds.), Handbook of schizophrenia: Vol. 5. Neuropsychology, psychophysiology and information processing (pp. 599-650). Amsterdam: Elsevier.

Herjanic, B., \& Campbell, W. (1977). Differentiating psychiatrically disturbed children on the basis of a structured interview. Journal of Abnormal Child Psychology, 5, 127-134.

Herjanic, B., \& Reich, W. (1982). Development of a structured psychiatric interview for children: Agreement between child and parent on individual symptoms. Journal of Abnormal Child Psychology, 10, $307-324$

Jacobsen, L. K., Hong, W. L., Hommer, D. W., Hamburger, S. D. Castellanos, F. X., Frazier, J. A., Giedd, J. N., Gordon, C. T., Karp, B. I., McKenna, K., \& Rapoport, J. L. (1996). Smooth pursuit eye movements in childhood onset schizophrenia: Comparison with attention-deficit hyperactivity disorder and normal controls. Biological Psychiatry, 40, 1144-1154

Karno, M., \& Norquist, G. S. (1989). Schizophrenia: Epidemiology. In H. I. Kaplan, \& B. J. Sacock (Eds.), Comprehensive textbook of psychiatry, Volume 1 (5th ed., pp. 699-705). Baltimore, MD: Williams \& Wilkins.

Karras, A. (1968). Choice reaction time of chronic and acute psychiatric patients under primary or secondary aversive stimulation. British Journal of Social and Clinical Psychology, 7, 270-279.

Karras, A. (1973). Effects of competing and complex responses on the reaction time of acute psychiatric groups. Joumal of Abnormal Psychology, 82, 134-138.

Kolvin, I., Ounsted, C., Humphrey, M., \& McNey, A. (1971). The phenomenology of childhood psychoses. British Joumal of Psychiatry, $118,385-395$.

Kramer, M. (1978). Population changes and schizophrenia, 1970-1985. In L. C. Wynne, R. L. Cromwell, \& S. Matthysse (Eds.), The nature of schizophrenia: New approaches to research and treatment (pp. 545-571). New York: Wiley.

Kristofferson, M. W. (1967). Shifting attention between modalities: A comparison of schizophrenics and normals. Journal of Abnormal Psychology, 72, 388-394.

McKenna, K., Gordon, C. T., Lenane, M. C., Kaysen, D., \& Rapoport, J. L. (1994). Looking for childhood-onset schizophrenia: The first 71 cases screened. Journal of the Academy of Child and Adolescent Psychiatry, 33, 636-644.

Miller, M. B., Chapman, L. J., Chapman, J. P., \& Kwapil, T. R. (1993). Slowness and the preceding preparatory interval effect in schizophrenia. Journal of Abnormal Psychology, 102, 145-151.

Niemi, P., \& Näätänen, R. (1981) . Foreperiod and simple reaction time Psychological Bulletin, 89, 133-162.

Nuechterlein, K. H. (1977). Reaction time and attention in schizophrenia: A critical evaluation of the data and theories. Schizophrenia Bulletin, 3, 373-428.

Öhman, A. (1979). The orienting response, attention and learning: An information-processing perspective. In H. D. Kimmel, E. H. van Olst, \& J. F. Orlebeke (Eds.), The orienting reflex in humans (pp. 443-472). Hillsdale, NJ: Erlbaum.

Orvaschell, H., Tabrizi, M. A., \& Chambers, W. (1980). Schedule for Affective Disorders and Schizophrenia for School-Age ChildrenEpidemiologic Version (Kiddie-SADS-E) (3rd ed.). New York: New 
York State Psychiatric Institute and Yale University School of Medicine

Overall, J. E., Hollister, L. E., \& Pichot, P. (1967). Major psychiatric disorders: A four dimensional model. Archives of General Psychiatry, 24, 146-151.

Posner, M. I., Nissen, M. J., \& Klein, R. M. (1976). Visual dominance: An information-processing account of its origins and significance. Psychological Review, 8.3, 157-171.

Reich, W., \& Welner, Z. (1988). Diagnostic Interview for Children and Adolescents-RC (DSM-IIl-R version, rev. version $V-R$ ). St. Louis, MO: Washington University.

Rist, F., \& Cohen, R. (1991). Sequential effects in the reaction times of schizophrenics: Crossover and modality shift effects. In S. R. Steinhauer, J. H. Gruzelier, \& J. Zubin (Eds.), Handbook of schizophrenia: Vol. 5. Neuropsychology, psychophysiology and information processing (pp. 241-271). Amsterdam: Elsevier.

Rosenbaum, G. (1967). Reaction time indices of schizophrenic motivation: A cross-cultural replication. British Journal of Psychiatry, 113, $537-541$.

Rutter, M. (1972). Childhood schizophrenia reconsidered. Journal of Autism and Childhood Schizophrenia, 2, 315-337.

Shakow, D. (1962). Segmental set: A theory of the formal psychological deficit in schizophrenia. Archives of General Psychiatry, 6, 1-17.

Spohn, H. E., \& Strauss, M. E. (1989). Relation of neuroleptic and anticholinergic medication to cognitive functions in schizophrenia. Journal of Abnormal Psychology, 98, 367-380.

Strandburg, R. J., Marsh, J. T., Brown, W. S., Asarnow, R. F., \& Guthrie, D. (1994). Information-processing deficits across childhood- and adult-onset schizophrenia. Schizophrenia Bulletin, 20, 685-695.

Sutton, S., Hakerem, G., Zubin, J., \& Portnoy, M. (1961). The effect of shift of sensory modality on serial reaction time: A comparison of schizophrenics and normals. Americal Journal of Psychology, 74, 224-232.

Sutton, S., \& Zubin, J. (1965). Effect of sequence on reaction time in schizophrenia. In A. T. Welford \& J. E. Birren (Eds.), Behavior, aging, and the nervous system (pp. 562-579). Springfield, IL: Charles C Thomas.

Tizard, J., \& Venables, P. H. (1956). Reaction time responses by schizophrenics, mental defectives, and normal adults. American Journal of Psychiatry, 112, 803-810.

Werry, J. S. (1992). Child and adolescent (early onset) schizophrenia: A review in light of DSM-III-R. Journal of Autism and Developmental Disorders, 22, 601-624.

Woodworth, R. S., \& Schlosberg, H. (1954). Experimental psychology (rev. ed.). New York: Henry Holt.

Zahn, T. P. (1970). Effects of reductions in uncertainty on reaction time in schizophrenic and normal subjects. Journal of Experimental Research in Personality, 4, 135-143.

Zahn, T. P. (1977). Comments on "Reaction time and attention in schizophrenia.' Schizophrenia Bulletin, 3, 452-456.

Zahn, T. P., \& Carpenter, W. T. (1978). Effects of short-term outcome and clinical improvement on reaction time in acute schizophrenia. Journal of Psychiatric Research, 14, 59-68.

Zahn, T. P., Jacobsen, L. K., Gordon, C. T., McKenna, K., Frazier, J. A., \& Rapoport, J. L. (1997). Autonomic nervous system markers of psychopathology in childhood onset schizophrenia. Archives of General Psychiatry, 54, 904-912.

Zahn, T. P., Pickar, D., \& Haier, R. J. (1994). Effects of clozapine, fluphenazine, and placebo on reaction time measures of attention and sensory dominance in schizophrenia. Schizophrenia Research, 13, $133-144$.

Zahn, T. P., Rosenthal, D., \& Shakow, D. (1961). Reaction time in schizophrenic and normal subjects in relation to the sequence of series of regular preparatory intervals. Journal of Abnormal and Social Psychology, 63, 161-168.

Zahn, T. P., Rosenthal, D., \& Shakow, D. (1963). Effects of irregular preparatory intervals on reaction time in schizophrenia. Journal of Abnomal and Social Psychology, 67, 44-52.

Received June 17, 1996

Revision received April 28, 1997

Accepted June 30, 1997 